\title{
La Familia Calyptraeidae en el Perú (Gastropoda: Caenogastropoda)
}

\section{The family Calyptraeidae in Peru (Gastropoda: Caenogastropoda)}

\section{Carlos Paredes y Franz Cardoso}

Universidad Nacional Mayor de San Marcos, Facultad de Ciencias Biológicas, Laboratorio de Biología y Sistemática de Invertebrados Marinos, Apdo. 110058, Lima 11, Perú.

y

Departamento de Malacología, Museo de Historia Natural, Universidad Nacional Mayor de San Marcos, Apdo. 14-0434, Lima 14, Perú.

E-mail: Carlos Paredes cparedesq@unmsm.edu.pe

\section{Resumen}

En el presente trabajo se realiza una revisión taxonómica de la familia Calyptraeidae Lamarck, 1809, en la costa Peruana. Se confirma la presencia de 17 especies de las 19 reportadas por diversos autores. Crepidula arenata (Broderip, 1834) es sinónimo de C. excavata (Broderip, 1834). Se da información sobre la distribución geográfica y algunas relaciones ecológicas.

Palabras claves: Mollusca, Gastropoda, Calyptraeidae, Taxonomía, Perú.

\section{Abstract}

In the present work the taxonomic of the family Calyptraeidae Lamarck, 1809 in the Peruvian coast are reviewed. The presence of 17 species of the 19 is confirmed reported by diverse authors. Crepidula arenata (Broderip, 1834) is synonymous of C. excavata (Broderip, 1834). Information on the distribution geographical and same relations ecological is presented.

Keywords: Mollusks, Gastropod, Calyptraeidae, Taxonomy, Perú.

\section{Introducción}

La familia Calyptraeidae Lamarck, 1809, incluye Caenogastropoda mayormente sedentarios, habitantes de los fondos rocosos desde la zona intermareal hasta el sublitoral en todos los mares del mundo, contando aproximadamente con 76 especies, de las cuales se han reportado 40 en el Pacífico Este, y 20 para el mar peruano (Paredes et al., 1999). Debido a su morfología relativamente simple y la gran plasticidad que poseen, se observa mucha variación en la forma de la conchilla, lo que ha ocasionado muchos casos de sinonimia. En años recientes se está considerando además la morfología de la rádula, la anatomía interna y los caracteres moleculares; con lo cual se viene dilucidando diversas interrogantes de carácter taxonómico (Simone, 2002; Collin, 2003a,b, 2005). El objetivo de este trabajo ha sido actualizar la taxonomía de la familia Calyptraeidae en el Perú, puesto que diferenciando claramente las especies, será posible estudiar su biología, ecología y aspectos aplicados, como ya está ocurriendo en Chile (Chaparro et al., 2002a, b; Montiel et al., 2005; Véliz et al., 2001, 2003).

\section{Material y metodos}

Se han examinado 1237 ejemplares de Calyptraeidae colectados a mano en la zona intermareal y el sublitoral rocoso de la costa peruana.

Para la determinación taxonómica se utilizó la bibliografía disponible, y para el ordenamiento sistemático se siguió a Collin et al. (2003a, b). El material está depositado en la colecciones del Laboratorio de Biología y Sistemática de Invertebrados Marinos de la Universidad Nacional Mayor de San Marcos (LaBSIM) y el Museo de Historia Natural de la Universidad Nacional Mayor de San Marcos (MUSM).

\section{Sistematica}

FAMILIA CALYPTRAEIDAE LAMARCK, 1809

GÉNERo CALYPTRAE $A$ LAMARCK, 1799

\section{CALYPTRAE A MAMILLARIS BRODERIP, 1834}

(Figura 1a)

Calyptraea mamillaris, Dall, 1909: 233; Peña, 1970: 159; Abbott, 1974: 139; Collin, 2003a: 550, fig. 1E; Collin, 2003b: 639.

Calyptraea (Calyptraea) mamillaris, Keen, 1971: 456, fig. 801; Alamo \& Valdivieso, 1987: 36, fig. 73; Cruz \& Jiménez, 1994:123, fig. 18; Hendrickx \& Toledano, 1994: 37; Paredes et al., 1999: 23; Skoglund, 2002: 71.

Concha cónica con ápex central y septo en forma de rampa espiral, abertura redondeada; sólo presenta estrías de crecimiento; coloración externa e interna blanca, brillante por dentro. Diámetro: $26,8 \mathrm{~mm}$.

Material examinado: 6 lotes, 12 ejemplares, LaBSIM, MUSM.

Hábitat: Fondo areno-fangoso sobre piedras pequeñas.

Localidades: Tumbes (Boca de Capones, Puerto Pizarro, Zorritos, Bocapán).

Distribución: Bahía Magdalena, Baja California a Paita Perú, (Keen, 1971), y hacia el sur hasta 7,5 millas SW de Isla Lobos de Afuera, Perú (Álamo \& Valdivieso, 1987).

\section{GÉNero Crepidul $A$ Lamarck, 1799}

Subgénero JANACUS Mörch, 1852

CRepidula (J.) LESSONII Broderip, 1834

(Figura 1b)

Crepidula lessonii, Peña, 1970: 159; Keen, 1971: 460, fig. 812; Alamo \& Valdivieso, 1987: 37, fig. 77; Hendrickx y Toledano, 1994: 38 Paredes et al., 1999: 23; Mogollón et al., 1999: 43, fig. 5g,h; Skoglund, 2002: 36

Crepidula (Crepidula) lessonii, Abbott, 1974: 142.

Crepidula (Janacus) lessoni, Collin, 2003a: 547; Collin, 2003b: 636.

Concha ovalada con ápex marginal o submarginal; la escultura consiste en láminas concéntricas; exterior e interior blanco, en algunos se observan bandas radiales pardo rojizas; el septo es blanco translúcido y lleva un suave lomo que termina en una escotadura central. Longitud: $28,3 \mathrm{~mm}$. 
Material examinado: 17 lotes, 120 ejemplares, LaBSIM, MUSM.

Hábitat: Intermareal rocoso.

Localidades: Tumbes (Estero de Zarumilla, Zorritos, Bocapán, El Rubio, Cancas, Punta Sal), Piura (Máncora, Los Órganos, Lobitos).

Distribución: Playa Delfín, Sinaloa, México (Hendrickx \& Toledano, 1994) a Zorritos, Perú (Collin, 2003b), y hacia el sur hasta la Isla Los Chimus, Perú (Mogóllon et al., 1999).

\section{Crepidula (J.) nivea C.B. Adams, 1852}

(Figura 1c)

Crepidula nivea, Paredes \& Cardoso, 1998: 119, fig. 1; Paredes et al. 1999: 23; Skoglund, 2002: 72.

Crepidula (Janacus) nivea, Collin, 2003b: 637.

Concha ovoide con perfil variable, ápex marginal, convexa o deprimida; escultura con líneas de crecimiento definidas, a veces levantadas como lamelas; periostraco amarillento; septo con suave escotadura central; interior aporcelanado brillante. Longitud: $20,8 \mathrm{~mm}$.

Material examinado: 4 lotes, 24 ejemplares, LaBSIM

Hábitat: Mediolitoral rocoso, en las depresiones de las rocas.

Localidades: Lima (Bahía de Ancón, Las Conchitas), Ica (Playa Atenas).

Distribución: Sur de California (Hoagland, 1977) a Puerto Pizarro, Perú (Collin, 2003b) y hacia el sur hasta Lima, Perú (Paredes \& Cardoso, 1998).

\section{Crepidula (J.) Striolata MEnKe, 1851}

(Figura 1d)

Crepidula striolata, Keen, 1971: 461, fig. 817; Abbott, 1974: 142; Alamo y Valdivieso, 1987: 37; Hendrickx \& Toledano, 1992: 38 Paredes et al., 1999: 23; Skoglund, 2002: 73.

Crepidula (Janacus) striolata, Collin, 2003a: 547; Collin, 2003b: 637

Concha ovalada y baja con el ápex submarginal; con líneas de crecimiento irregulares y 2 ó 3 costillas radiales suaves; el periostraco es hirsuto y amarillento; coloración blanco amarillento con bandas radiales parduscas y una tonalidad pardusca cerca del ápex; el septo es blanco, algo hundido en el lado izquierdo. Longitud: $38,7 \mathrm{~mm}$.

Material examinado: 5 lotes, 7 ejemplares, LaBSIM, MUSM.

Hábitat: Mediolitoral rocoso.

Localidades: Tumbes (Bocapán), Piura (Máncora, Los Órganos, Bahía de Sechura, Parachique).

Distribución: San Diego, California (Hoagland, 1977) a Río Mar, Panamá (Collin, 2003a), y probablemente al sur hasta San Bartolo, Lima, Perú (Álamo \& Valdivieso, 1987).

Observaciones: Se le encuentra también como epizoico sobre Argopecten purpuratus.

\section{GÉNERo CREPIDULA S.L.}

\section{CREPIDUla EXCAVATA (Broderip, 1834)}

\section{(Figura 1e)}

Crepidula excavata, Dall, 1909:234; Peña, 1970: 159; Keen, 1971 460, fig. 810; Abbott, 1974: 142, fig. 1561; Alamo \& Valdivieso, 1987: 37; Hendrickx \& Toledano, 1994: 38; Paredes et al., 1999: 23;
Skoglund, 2002: 72; Collin, 2003a: 548, fig. 9B; Collin, 2003b: 637. Crepidula arenata, Keen, 1971: 460, fig.809; Abbott, 1974: 142; Hendrickx \& Toledano, 1994: 38; Skoglund, 2002: 72; Collin, 2003a: 548; Collin, 2003b: 637.

Concha ovalada con el ápex posterior y lateral, escultura con líneas de crecimiento, y a veces con el periostraco adherente; color externo blanco amarillento y pardo brillante por dentro, con el septo blanquecino. Longitud: 39,6 $\mathrm{mm}$.

Material examinado: 16 lotes, 118 ejemplares, LaBSIM, MUSM.

Hábitat: Infralitoral rocoso.

Localidades: Tumbes (Puerto Pizarro, Bocapán, Cancas), Piura (Máncora, Los Órganos, Playa San Pedro, Paita, Bahía de Sechura, Punta Aguja, Bayovar).

Distribución: Bahía Magdalena, Baja California Sur, México a Puerto Pizarro, Perú (Collin, 2003b), y al sur hasta Chile (Hoagland, 1977).

\section{CREPIDUla INCURVA (Broderip, 1834)}

(Figura 1f)

Crepidula incurva, Dall, 1909: 234; Peña, 1970: 159; Keen, 1971: 460, fig. 811; Álamo \& Valdivieso, 1987: 37, fig. 76; Hendrickx \& Toledano, 1994: 38; Paredes et al., 1999: 23; Skoglund, 2002: 72; Collin, 2003a: 548, Collin, 2003b: 637 .

Crepidula (Crepidula) incurva, Abbott, 1974: 142.

Concha subrectangular con el ápex semejando una copa que sobrepasa el margen; escultura consiste en costillas longitudinales, color externo marrón oscuro, interior brillante y casi negro, el septo es blanco. Longitud: $12,7 \mathrm{~mm}$.

Material examinado: 12 lotes, 72 ejemplares, LaBSIM, MUSM.

Hábitat: Infralitoral rocoso.

Localidades: Tumbes (Puerto Pizarro, El Rubio, Bocapán), Piura (Máncora, Los Órganos, Paita, Punta Aguja).

Distribución: San Felipe, Baja California, México a Paita, Perú (Hoagland, 1977).

\section{Crepidula onyX Sowerby, 1824}

\section{(Figura 1g)}

Crepidula onyx, Dall, 1909: 174, 234; McLean, 1969: 36-37, fig. 18.5; Peña, 1970: 159; Keen, 1971: 460, fig. 814; Abbott, 1974: 141, fig. 1560; Alamo \& Valdivieso, 1987: 37; Hendrickx \& Toledano, 1994: 38; Paredes et al., 1999: 23; Skoglund, 2002: 73; Collin, 2003a: 549; Collin, 2003b: 638.

Concha ovalada con el ápex muy cerca del margen; escultura concéntrica con el periostraco hirsuto y muy adherente; color externo pardo, interior salmón oscuro brillante y el septo blanco, ligeramente cóncavo con dos curvaturas separadas por una ligera escotadura central. Longitud: $28,4 \mathrm{~mm}$.

\section{Material examinado: 3 lotes, 3 ejemplares, LaBSIM}

Hábitat: Epizoico en la concha de abánico Argopecten purpuratus.

Localidades: Tumbes (Zorritos), Piura (Bahía de Sechura), Lima (Isla Pachacamac).

Distribución: Santa Barbara, California a Venado, Panamá (Collin, 2003a), y hacia el sur hasta Cancas, Perú (Peña, 1970). También ocurre en Playa Orengo, Argentina (Collin, 2003a). 


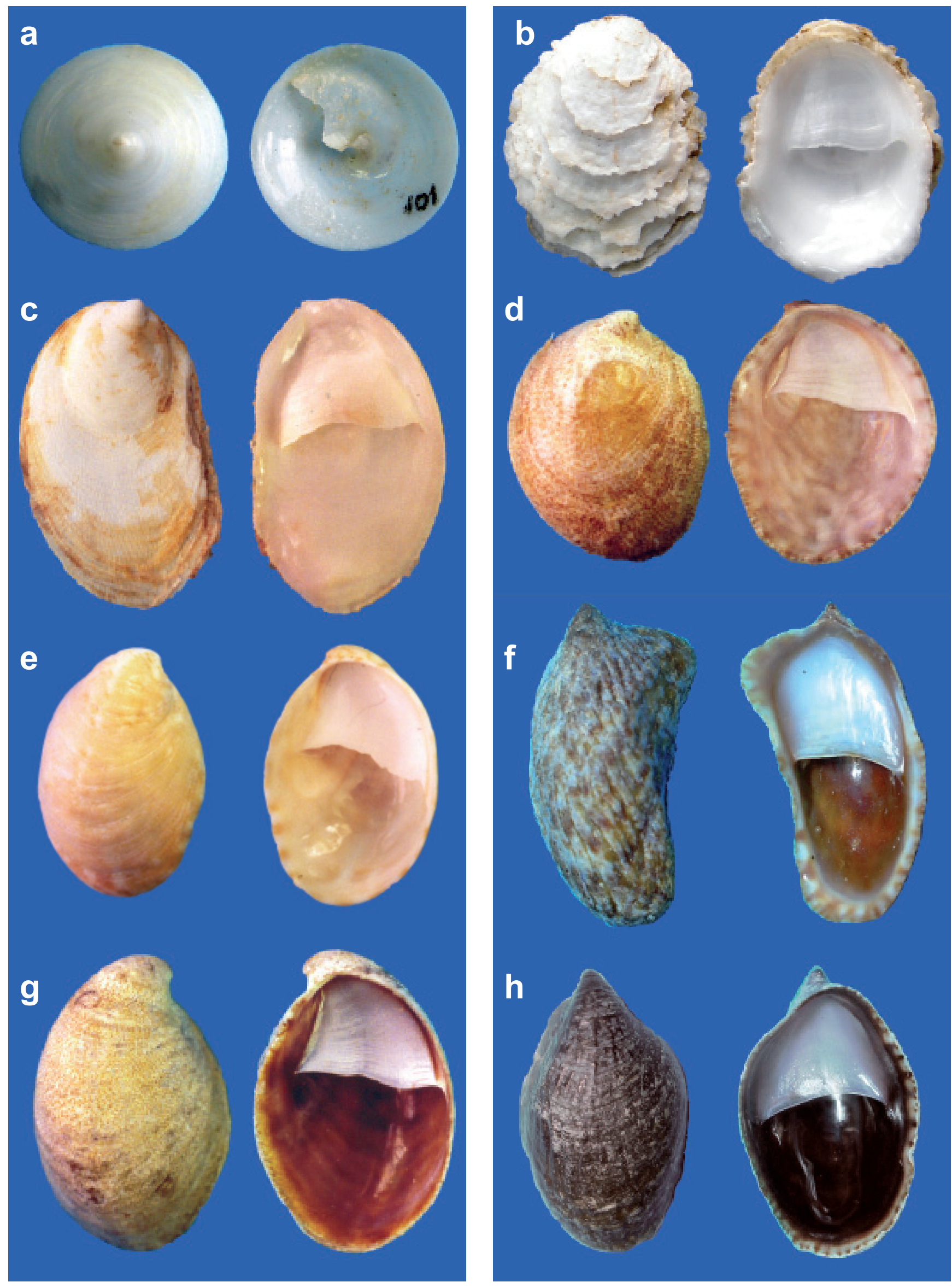

Figura 1. (a) Calyptraea mamilaris, diámetro 26,8 mm; (b) Crepidula (J.) lessonii, longitud 28,3 mm; (c) Crepidula (J.) nivea, longitud 20,8 mm; (d) Crepidula (J.) striolata, longitud 38,7 mm; (e) Crepidula excavata, longitud 39,6 mm; (f) Crepidula incurva, longitud 28,4 mm; (g) Crepidula onyx, longitud 9,9 mm; (h) Crepidula rostrata, longitud 12,5 mm. 

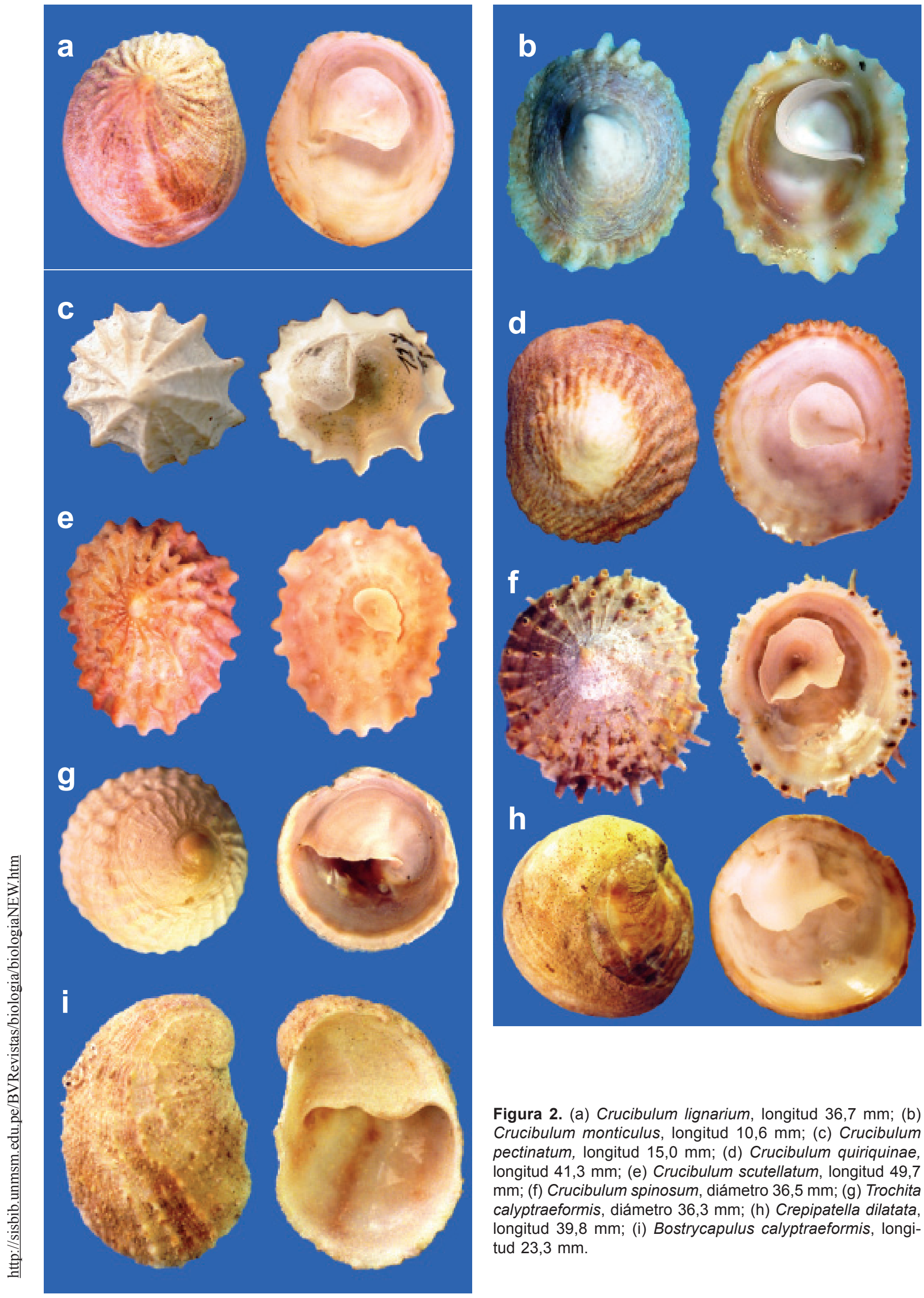

Figura 2. (a) Crucibulum lignarium, longitud $36,7 \mathrm{~mm}$; (b) Crucibulum monticulus, longitud 10,6 mm; (c) Crucibulum pectinatum, longitud 15,0 mm; (d) Crucibulum quiriquinae, longitud 41,3 mm; (e) Crucibulum scutellatum, longitud 49,7 mm; (f) Crucibulum spinosum, diámetro 36,5 mm; (g) Trochita calyptraeformis, diámetro $36,3 \mathrm{~mm}$; (h) Crepipatella dilatata, longitud 39,8 mm; (i) Bostrycapulus calyptraeformis, longitud $23,3 \mathrm{~mm}$. 


\section{Crepidula rostrata C. B. Adams, 1852}

(Figura 1h)

Crepidula rostrata, Peña, 1970: 159; Keen, 1971: 461, fig. 816; Alamo \& Valdivieso, 1987: 37; Paredes et al., 1999: 23; Skoglund, 2002: 73.

Similar a $C$. incurva, pero el ápex se levanta en forma recta sobre el margen; las costillas son más gruesas que C. incurva; coloración marrón oscuro y el septo tiene el borde recto o ligeramente cóncavo. Longitud: $12,5 \mathrm{~mm}$.

Material examinado: 9 lotes, 19 ejemplares, LaBSIM, MUSM.

Hábitat: Sobre las raíces de los mangles.

Localidades: Tumbes (Bocapán), Piura (Máncora, Los Cangrejos).

Distribución: Bahía San Carlos, Sonora, México (DuShane \& Poorman, 1967) a Puerto Pizarro, Tumbes, Perú (Peña, 1970).

Observaciones: Las diferencias morfológicas de la conchilla con respecto a Crepidula incurva no son muy relevantes, y podría ser un caso de sinonimia.

\section{Género Crucibulum Schumacher, 1817 Crucibulum lignarium (Broderip, 1834)} (Figura 2a)

Crucibulum (Crucibulum) lignarium, Keen, 1971: 463, fig. 822; Peña, 1976: 2, fig. 3; Alamo \& Valdivieso, 1987: 41, fig. 80; Paredes et al., 1999: 23; Mogollón et al., 1999: 44, fig. 2m,n; Skoglund, 2002: 73.

Crucibulum lignarium, Abbott, 1974: 140; Hendrickx \& Toledano, 1994: 39; Collin, 2003b: 638.

Concha cónica trigonal o redondeada, el ápex en el tercio posterior; escultura presenta finas costillas radiales las que son más gruesas en la parte posterior bajo el ápex; color externo pardo oliváceo con bandas radiales blancas, interior brillante y blanquecino; el septo es blanco y puede estar abierto en el lado de la fijación. Longitud: $36,7 \mathrm{~mm}$

Material examinado: 4 lotes, 6 ejemplares, LaBSIM

Hábitat: Intermareal rocoso.

Localidades: Tumbes (Puerto Pizarro), Piura (Isla Lobos de Tierra), Lima (Bahía de Ancón), Callao (Isla San Lorenzo).

Distribución: Bahía Santa Maria, Sinaloa, México (Hendrickx \& Toledano, 1994) a Ancud, Chiloe, Chile (Collin, 2003b).

\section{Crucibulum monticulus Berry, 1969}

(Figura 2b)

Crucibulum (Crucibulum) monticulus, Keen, 1971: 463, fig. 823 Abbott, 1974: 140; Alamo \& Valdivieso, 1987: 41, fig. 81; Skoglund 1992: 37; Hendrickx \& Toledano, 1994: 39; Paredes et al., 1999: 23; Skoglund, 2002: 73-74.

Concha débil de contorno oval con el ápex subcentral y curvado hacia el lado derecho; escultura presenta costillas radiales, las cuales dan un aspecto aserrado al margen; el septo se fija en el lado derecho más ampliamente que en las otras especies del subgénero; coloración externa e interna blanquecina con manchas pardas en el interior, el septo es blanco. Longitud: 20,7 mm.

Material examinado: 4 lotes, 4 ejemplares, LaBSIM.
Hábitat: Fondo rocoso, 7-11 m de profundidad.

Localidades: Tumbes (Boca de Capones, Bocapán), Callao (Isla San Lorenzo), Ica (Bahía Independencia).

Distribución: Isla Smith, Bahía de los Angeles, Baja California, México (Skoglund, 1988) a Islas Guañape, La Libertad, Perú (Alamo \& Valdivieso, 1987).

\section{Crucibulum pectinatum Carpenter, 1856}

(Figura 2c)

Crucibulum (Dispotaea) pectinatum, Keen, 1971: 465, fig. 829; Alamo \& Valdivieso, 1987: 42; Hendrickx \& Toledano, 1994: 39; Paredes et al., 1999: 23; Skoglund, 2002: 74.

Concha delgada con la base oval o redondeada con ápex subcentral; escultura presenta 14 costillas radiales que se proyectan más allá del borde de la conchilla; en la zona del ápex se observan las líneas de crecimiento, las cuales hacia los márgenes se levantan entre las costillas para formar profundos hoyos; por lo que la escultura parece cancelada. El color de la conchilla tanto externo como interno es crema opaco; el septo es deprimido anteriormente, está ampliamente fijado al lado posterior de la conchilla y tiene la misma coloración opaca. Longitud: $15,0 \mathrm{~mm}$.

Material examinado: 1 lote, 1 ejemplar, LaBSIM.

Hábitat: Varado en la playa arenosa.

Localidades: Tumbes (El Bendito).

Distribución: Bahía de Guaymas, Sonora, México a Perú (Keen, 1971).

\section{CRUCIBULUM QUIRIQUINAE (LESSON, 1830)}

$$
\text { (Figura 2d) }
$$

Crucibulum quiriquinae, Dall, 1909: 233; Keen, 1971: 463; Simone, 2002: 51 , figs. $18,19,68,69,207-226$.

Crucibulum (Crucibulum) quiriquinae, Marincovich, 1973: 32, fig 67; Alamo \& Valdivieso, 1987: 41; Guzmán et al., 1998: 43-44, fig. 39; Paredes et al., 1999: 23

Concha cónica con ápex casi central; escultura consiste en suaves costillas radiales que se proyectan en la base dándole el aspecto crenulado, se observa también que algunas costillas no son radiales sino que se dirigen formando arcos entre los bordes por debajo de la zona apical; el septo tiene forma de copa y es de color blanco; color externo blanco amarillento con las costillas de color pardo, el interior de la concha es banco brillante y presenta una banda parda periférica. Diámetro: $46,1 \mathrm{~mm}$.

Material examinado: 16 lotes, 48 ejemplares, LaBSIM, MUSM.

Hábitat: Infralitoral rocoso.

Localidades: Lima (Bahía de Ancón, La Conchitas, Lurin, Pucusana), Callao (Isla San Lorenzo), Ica (Pisco, Laguna Grande, Lagunillas, Bahía Independencia).

Distribución: Pucusana, Lima, Perú a Concepción, Chile

(Marincovich, 1973).

Observaciones: Véliz et al (2001) sostienen que esta especie presenta una marcada variación morfológica de acuerdo al sustrato donde habita, lo cual puede afectar las características reproductivas. 


\section{Crucibulum scutellatum (Wood, 1828)}

(Figura 2e)

Crucibulum imbricatum, Dall, 1909: 173, 233.

Crucibulum scutellatum, Peña, 1970: 160; Abbott, 1974: 140; Hendrickx \& Toledano, 1994: 40; Collin, 2003a: 549; Collin, 2003b: 638.

Crucibulum (Crucibulum) scutellatum, Keen, 1971: 463, fig. 825; Alamo \& Valdivieso, 1987: 41, fig. 82; Paredes et al., 1999: 23; Skoglund, 2002: 74.

Concha en forma de cono bajo con el ápex subcentral; escultura con costillas radiales gruesas y escamosas que pueden estar entrelazadas por escultura concéntrica, y se proyecta en el borde de la concha con espinas gruesas; el septo en forma de copa se fija al ápex y por uno de sus lados a la concha; coloración pardusca con el septo blanco: Longitud: 67,0 $\mathrm{mm}$.

Material examinado: 23 lotes, 72 ejemplares, LaBSIM, MUSM.

Hábitat: Infralitoral rocoso.

Localidades: Tumbes (Puerto Pizarro, Zorritos, Bocapán, Cancas, Punta Sal), Piura (Máncora, Los Órganos, Lobitos, Paita, Bocana de San Pedro, Bayovar, Islas Lobos de Tierra), Ica (Bahía Independencia).

Distribución: Punta Piaxtla, Sinaloa, México (Hendrickx \& Toledano, 1994) a Ecuador (Keen, 1971) y al sur hasta Pimentel, Lambayeque, Perú (Peña, 1970).

\section{CRUCibulum SPINosum (Sowerby, 1824)}

\section{(Figura 2f)}

Crucibulum spinosum, Dall, 1909: 173, 233; McLean, 1969: 37, fig 18.8; Peña, 1970: 160; Abbott, 1974: 140, fig. 1542; Hendrickx \& Toledano, 1994: 40; Collin, 2003a: 549, fig. 2D; Collin, 2003b: 638 . Crucibulum (Crucibulum) spinosum, Keen, 1971: 463, fig. 826; Álamo \& Valdivieso, 1987: 42, fig. 83; Paredes et al., 1999: 23; Mogollón et al., 1999: 44-45, fig. 21, 6 k; Skoglund, 2002: 74.

Concha en forma de cono bajo con el ápex ligeramente posterior y cuya punta esta curvada hacia el lado derecho; escultura presenta costillas radiales de las que se levantan en forma intercalada espinas tubulares; coloración general pardo amarillento con el septo blanco. Diámetro: 46,1 $\mathrm{mm}$.

Material examinado: 27 lotes, 206 ejemplares, LaBSIM, MUSM.

Hábitat: Infralitoral areno-pedregoso.

Localidades: Tumbes (Cancas, Bocapán), Piura (Máncora, Lobitos, Paita, Bayovar, Islas Lobos de Tierra), Ancash (Tortugas, Los Chimus, Samanco, Casma), Ica (Laguna Grande, Lagunillas, Bahía Independencia).

Distribución: Estero El Verde, Sinaloa, México (Hendrickx \& Toledano, 1994) a Santa María, Lima, Perú (Collin, 2003a), y hacia el sur a Tomé, Chile (Keen, 1971).

\section{GÉnero Trochita Schumacher, 1817}

\section{TROCHITA CALYPTRAEFORMIS (BORN, 1778)} (Figura 2g)

Trochita trochiformis, Dall, 1909: 175, 233, pl 23, fig. 1; Abbott, 1974 143; Reid \& Osorio, 2000: 124, fig. 3H, I; Simone, 2002: 55, 60-61, figs. $20,21,72,73,227-251$.

Calyptraea (Trochita) trochiformis, Peña, 1970: 159; Keen, 1971: 456, fig. 804; Marincovich, 1973: 31-32, fig. 65; Álamo \& Valdivieso, 1987: 36, fig. 74; Guzmán et al., 1998: 41-42, fig. 35; Paredes et al., 1999: 23; Skoglund, 2002: 72.

Trochita Calyptraeformis, Collin, 2003a: 550, fig. 2l, 9C; Collin, 2003 b: 639.

Concha cónica de altura variable y las vueltas algo infladas, ápex subcentral; escultura consiste en costillas oblicuas; septo corto en forma de rampa espiral; color externo blanquecino con periostraco hirsuto y adherente, el interior es marrón claro y el septo blanco. Diámetro: $36,3 \mathrm{~mm}$.

Material examinado: 34 lotes, 158 ejemplares, LaBSIM, MUSM.

Hábitat: Fondo rocoso y pedregoso en el infralitoral.

Localidades: Lima (Bahía de Ancón, Las Conchitas, Punta Roquitas-Miraflores, Isla Pachacamac, Pucusana), Ica (Pisco, Paracas, Mendieta, Playa Atenas, Laguna Grande, Lagunillas, Bahía Independencia), Arequipa (Chala), Moquegua (Ilo, Playa tres Hermanas), Tacna (Vila Vila).

Distribución: Playa Besique, Ancash, Perú (Peña, 1970) a Bahía de Herradura, Chile (Collin, 2003a), y al sur hasta la Isleta Huemules, Estero Elefantes, Chile (Reid \& Osorio, 2000). También ocurre en el Oeste de África (Keen \& Coan, 1975).

Observaciones: Simone (2002), siguiendo a Keen (1971) la cita para Manta, Ecuador, pero sin revisar material. Se ha observado al cangrejito comensal Pinnotheres ostreum Say, 1817 viviendo en la cavidad paleal.

\section{GÉnero CRePipatella Lesson, 1830}

\section{CREPIPATELLA DILATATA (LAMARCK, 1822)}

(Figura 2h)

Crepidula dilatata, Dall, 1909: 174, 234; Peña, 1970: 159; Hoagland, 1977: 372; Guzmán et al., 1998: 42, fig. 36; Reid \& Osorio, 2000: 123-124, fig. 3f, $g$.

Crepipatella dilatata, Dell, 1971: 205-206; Marincovich, 1973: 32, fig. 66; Álamo \& Valdivieso, 1987: 41, fig. 79; Paredes et al., 1999: 23; Mogollón et al., 1999: 43-44, fig. 5i, j; Skoglund, 2002: 73; Collin, 2003a: 546, 3C; Collin, 2003b: 636

Concha baja o alta, cóncava, ovalada o redondeada, el ápex es pronunciado y orientado al lado derecho; escultura con finas líneas radiales de color pardo y también se notan las líneas de crecimiento; el septo es cóncavo y presenta más de la mitad del borde izquierdo libre; el color externo pardo claro rojizo; interior blanco brillante con una banda de color pardo en el margen. Longitud: $39,8 \mathrm{~mm}$.

Material examinado: 51 lotes, 251 ejemplares, LaBSIM, MUSM.

Hábitat: Mediolitoral e infralitoral rocoso, y epizoico en Aulacomya atra.

Localidades: Piura (Máncora), Lambayeque (Pimentel), La Libertad (Pacasmayo), Ancash (Chimbote, Tortugas), Lima (Huacho, Bahía de Ancón, Las Conchitas, Playa Ventanilla, La Herradura, Punta Hermosa, Isla Pachacamac, Pucusana), Callao (Isla San Lorenzo), Ica (El Chaco, Paracas, Playa Atenas, Laguna Grande, Lagunillas, Bahía Independencia), Arequipa (Chala), Moquegua (Ilo, Playa tres Hermanas).

Distribución: Isla San Lorenzo, Perú a Punta Arenas, Chile (Marincovich, 1973), más al norte hasta Pimentel, Perú (Peña, 1970). También ocurre en las Islas Malvinas, Argentina (Dell, 1971). 
Observaciones: La especie chilena Crepipatella fecunda Gallardo ,1979 es muy similar morfológicamente y solo difiere por el modo de reproducción, por lo que Reid \& Osorio (1979) sostienen que es sinónimo de $C$. dilatata. Se ha observado al cangrejito comensal Pinnotheres ostreum Say, 1817 viviendo en la cavidad paleal.

\section{Género Bostrycapulus Olsson \& Harbison, 1953}

\section{Bostrycapulus CALYPTRAEFORMIS (DESHAYES, 1830)}

(Figura 2i)

Crepidula aculeata, Dall, 1909: 233; McLean, 1969: 35-36, fig. 18.1; Peña, 1970: 159; Keen, 1971: 458, fig. 808; Abbott, 1974: 142; Alamo \& Valdivieso, 1987: 37, fig 75; Hendrickx \& Toledano, 1994: 37; Paredes et al., 1999: 23; Skoglund, 2002: 72; Collin, 2003: 543. Bostrycapulus aculeatus, Simone, 2002 [in part]: 18. Bostrycapulalus calyptraeformis, Collin, 2005: 94-95, fig. 1A, 5B, 5E, 8A.

Concha ovalada con el ápex posterior y lateral, septo plano y con un lomo mediano suave; escultura presenta hileras espirales de espinas cuyo grosor es variable; coloración pardusca con el septo blanco. Longitud: 23,3 mm.

Material examinado: 25 lotes, 116 ejemplares, LaBSIM, MUSM.

Hábitat: Mediolitoral e infralitoral rocoso; epizoico sobre otros moluscos.

Localidades: Tumbes (El Rubio, Bocapán, Cancas), Piura (Máncora, Los Órganos, Cabo Blanco, Los Cangrejos, Paita, Bahía de Sechura, Punta Aguja, Isla Lobos de Tierra).

Distribución: Playa Venado e Islas Perlas, Panamá, a Paita, Perú (Collin, 2005).

Observaciones: Collin (2005), señala que probablemente esta especie fue introducida en Hawaii, y Guam. Asimismo, también indica que los ejemplares de Perú, y Panamá y Hawaii difieren genéticamente pudiendo ser dos especies diferentes.

\section{Agradecimientos}

Los trabajos de campo fueron financiados por el Proyecto CSI Nº51001111 y el Proyecto CENSOR-UNMSM.

\section{Literatua citada}

Abbott, R.T. 1974. American Sheashells. The marine Mollusca of the Atlantic and Pacific coasts of north America. 2th. ed. Van Nostrand Reinhold Company. New York. 663 pp.

Alamo, V. \& V. Valdivieso. 1987. Lista sistemática de moluscos marinos del Perú. Bol. Inst. Mar. Perú. Vol. extraordinario: $1-205$.

Chaparro, O.R., J.L. Charpentier \& R. Collin. 2002a. Embryonic velar structure and function of two sibling species of Crepidula with different modes of development. Biol. Bull. 203: 80-86

Chaparro, O.R., R.J. Thompson \& S.V. Pereda. 2002b. Feeding mechanisms in the gastropod Crepidula fecunda. Mar. Ecol. Prog. Ser. 234: 171-181.

Collin, R. 2003a. The utility of morphological characters in gastropod phylogenetics: an example from the Calyptraeidae. Biological Journal of the Linnean Society 78: 541-593.

Collin, R. 2003b. Phylogenetic relationships among Calyptraeid gastropods and their implications for the biogeography of marine speciation. Syst. Biol. 52(5): 618-640.
Collin, R. 2005. Development, phylogeny, and taxonomy of Bostrycapulus (Caenogastropoda: Calyptraeidae), an ancient cryptic radiation. Zoological Journal of the Linnean Society 144: 75-101.

Dall, W.H. 1909. Report on a collection of shells from Peru, with a summary of the littoral marine mollusca of the Peruvian Zoological Province. Proc. U.S.N. Mus. 37(1704): 147294.

Dell, R.K. 1971. The marine mollusca of the Royal Society Expedition to southern Chile (1958-1959). Rec. Dominion Mus. (Wellington), 7(17): 155-233.

DuShane, H. \& R. Poorman. 1967. A checklist of mollusks for Guaymas, Sonora, México. The Veliger 9(4): 413-441.

Guzman, N., S. SAA \& L. Ortlieb. 1998. Catálogo descriptivo de los moluscos litorales (Gastropoda y Pelecypoda) de la zona de Antofagasta, $23^{\circ} \mathrm{S}$ (Chile). Estud. Oceanol. 17: 17-86.

Hendrickx, M.E. \& A. Toledano. 1994. Catálogo de Moluscos pelecypodos, gasterópodos y poliplacoforos. Colección de referencia, Estación Mazatlán, ICML, UNAM. Comisión Nacional para el Conocimiento y Uso de la Biodiversidad e Inst. Cienc. Mar y Limnol., UNAM, México. $71 \mathrm{pp}$.

Hoagland, K.E. 1977. Systematic review of fossil and recent Crepidula and discussion of the evolution of the Calyptraeidae. Malacologia 16(2): 353-420.

Keen, A.M. 1971. The shells of tropical West America. 2th. ed. Stanford Univ. Press, California. 1064 pp.

Keen, A.M. \& E.V. COAN. 1975. "Sea shells of tropical west America": additions and corrections to 1975. The Western Society of Malacologists Occasional Paper 1: 1-66.

Marincovich, L. 1973. Intertidal Mollusks of Iquique, Chile. Natural History Museum of Los Angeles County, Science Bulletin 16: 1-49.

McLean, J.H. 1969. Marine shells of Southern California. Science Series 24, Zoology $\mathrm{N}^{\circ} 11:$ 1-104.

Mogollon, V., N. Chirichigno, L. Clemente \& M. Peña. 1999. Gasterópodos (Mollusca) de Isla Los Chimus y alrededores (Santa, Ancash, Perú). Wiñay Yachay 3(2): 35-75.

Montiel, Y.A., O.R. Chaparro \& C.J. Segura. 2005. Changes in feeding mechanisms during early ontogeny in juveniles of Crepidula fecunda (Gastropoda, Calyptraeidae). Marine Biology 147: 1333-1342.

Paredes, C. \& F. Cardoso. 1998. Nuevos registros de gasterópodos para el litoral Peruano. Revista Peruana de Biología 5(2): 118-122.

Paredes, C., P. Huaman, F. Cardoso, R. Vivar \& V. Vera. 1999. Estado actual del conocimiento de los Moluscos acuáticos en el Perú. Rev. peru. biol. 6(1): 5-47.

Peña, M. 1970. Zonas de distribución de los gasterópodos marinos del Perú. Anales Científicos de la Universidad Agraria 8(3/ 4): $153-170$.

Peña, M. 1976. Registros adicionales de gasterópodos marinos del Perú. Anales científicos UNA 14(1/4): 1-8.

Reid, D.G. \& C. Osorio. 2000. The shallow-water marine Mollusca of the Estero Elefantes and Laguna San Rafael, southern Chile. Bull. Nat. Hist. Mus. Lond. (Zool.) 66(2): 109146.

Simone, L.R. 2002. Comparative morphological study and phylogeny of representatives of the superfamily calyptraeoidea (including Hipponicoidea) (Mollusca, Caenogastropoda). BiotaNeotropica 2(2): 1-137.

Skoglund, C. 1988. Deep water shells from off Isla Smith, Bahia de los Angeles, Baja California, México. The Festivus 20(11): 110-116. 
Skoglund, C. 2002. Panamic Province Molluscan literature. Additions and changes from 1971 through 2001. III Gastropoda The Festivus 33 (Supplement): 1-286.

Veliz, D., C. Guisado \& F.M. Winkler. 2001. Morphological, reproductive, and genetic variability among three populations of Crucibulum quiriquinae (Gastropoda: Calyptraeidae) in northern Chile. Marine Biology 139: 527-534.
Veliz, D., F.M. Winkler \& C. Guisado. 2003. Developmental and genetic evidence for the existence of three morphologically cryptic species of Crepidula in northern Chile. Marine Biology 143: 131-142. 